\title{
VIEWPOINT
}

\section{The many faces of nitric oxide: cytotoxic, cytoprotective or both}

\author{
J. W. WILEY \\ Department of Internal Medicine and General Clinical Research Center, University of Michigan Health System, Ann Arbor, MI, \\ USA
}

\begin{abstract}
Nitric oxide (NO) has emerged as a major modulator of cellular function in health and disease. In addition to its well-known role as a mediator of smooth muscle relaxation, a rapidly developing body of research suggests, paradoxically, that NO can have both cytotoxic and cytoprotective effects. In this issue of Neurogastroenterology and Motility, Choi et al. provide evidence that supports NO has a prosurvival effect on interstitial cells of Cajal in the mouse stomach. The objective of this short review is to place this interesting report in the context of the current literature.
\end{abstract}

Keywords carbon monoxide, hemeoxygenase, interstitial cells of Cajal, neuronal nitric oxide synthase, nitric oxide.

\section{INTRODUCTION}

The paradigm shifting discovery that mammalian cells have the capability of synthesizing the free radical nitric oxide (NO) has led to an explosion of research in the past decade to elucidate the role of this molecule under physiological and pathophysiological circumstances. NO has emerged as a fundamental signaling molecule regulating virtually every critical cellular function, and a potent mediator of cellular damage in a wide range of conditions. ${ }^{1} \mathrm{NO}$ has been implicated in both anti- and pro-apoptotic pathways depending on the cell type and conditions.

Address for correspondence

John W. Wiley, Department of Internal Medicine, University of Michigan Health System, MI, 48109, USA.

Tel: +1 734936 8080; fax: +1 734936 4024;

e-mail: jwiley@umich.edu

Received: 1 April 2007

Accepted for publication: 25 April 2007
At high concentrations, NO induces cell death during ischemic injury or as a consequence of neurodegenerative diseases. ${ }^{2,3}$ At lower concentrations, NO appears to be cytoprotective in neuronal cell lines. ${ }^{4,5}$ The cytotoxicity attributed to NO involves the generation of peroxynitrite, produced from the diffusion-controlled reaction between NO and another free radical, the superoxide anion. Peroxynitrite interacts with lipids, DNA, and proteins via direct oxidative reactions or via indirect, radical-mediated mechanisms. These reactions trigger cellular responses ranging from subtle modulations of cell signaling to overwhelming oxidative injury, committing cells to necrosis or apoptosis. Peroxynitrite generation appears to play a crucial pathogenic mechanism in conditions such as stroke, myocardial infarction, chronic heart failure, diabetes, circulatory shock, chronic inflammatory diseases, cancer, and neurodegenerative disorders. The neuroprotective effect of NO appears to be mediated at least in part by a cyclic GMP/protein kinase G (PKG) pathway. ${ }^{6,7}$ NO activates soluble guanylyl cyclase, an enzyme that catalyzes synthesis of cGMP from GTP. The mechanisms of PKG-mediated antiapoptotic actions are an active area of investigation. It is apparent that modulation of these pathways has important therapeutic implications.

Under physiologic conditions NO has a well-described function as an inhibitory neuromodulator in the nervous system and mediator of smooth muscle relaxation in the cardiovascular system and gastrointestinal tract. In this issue of Neurogastroenterology and Motility Choi et al. continue the expansion of our understanding of the many faces of $\mathrm{NO}^{\prime}$ s function. ${ }^{8}$ The factors underlying the survival and maintenance of interstitial cells of Cajal (ICC) are poorly understood. A robust literature supports the role for ICC as 
pacemaker cells in the gut and consequentially their pivotal role in regulating gastrointestinal motility. ${ }^{9,10}$ Loss of ICC is often associated with loss of neuronal nitric oxide synthase (nNOS) in humans suggesting a possible link. The aim of this study was to determine the effect of neuronal NO on ICC in the mouse gastric body. The volumes of ICC were determined in the gastric body and in organotypic cultures using immunohistochemistry, laser scanning confocal microscopy and three-dimensional reconstruction in transgenic mice that do not express nNOS ${ }^{-1-}$ and control mice. Functionally, the nNOS ${ }^{-/}$mouse demonstrates delayed gastric emptying because of impaired pyloric relaxation and diminished gastric motility. ${ }^{11}$ ICC numbers were determined in primary cell cultures after treatment with a NO donor or a NOS inhibitor. The volumes of myenteric c-Kit immunoreactive (a well-accepted marker for ICC) networks of ICC from $\mathrm{nNOS}^{-/-}$mice were significantly reduced compared with control mice. No significant differences in the volumes of c-Kit positive ICC were observed in the longitudinal muscle layers. ICC volumes were either decreased or unaltered in the circular muscle layer after normalization for volume of circular smooth muscle. Importantly, the number of ICC was increased after incubation with S-nitroso- $N$-acetylpenicillamine (NO donor) and decreased by $\mathrm{N}(\mathrm{G})$-nitro-L-arginine (inhibitor of NOS). The authors conclude that neuronally derived NO modulates ICC numbers and network volume in the mouse gastric body. Thus, in this mouse model NO appears to be a prosurvival factor for ICC.

This paper is noteworthy for several reasons. First, this is one of the first reports using a knockout mouse model for nNOS to examine the relationship between NO and ICC networks in the GI tract. Secondly, the authors have developed a method to assess cell volume and applied this to assess how the absence of neuronally derived NO influences ICC network volume in the mouse stomach. This represents a significant addition to historic morphometric techniques. It is relevant that his approach is generalizable to other cell systems and will likely be a more sensitive method to detect changes in cell volume when compared with conventional morphometric approaches. Thirdly, the authors' data suggests that there are significant regional differences in ICC numbers and networks in the mouse stomach. This establishes the need for a systematic assessment of ICC networks in other regions of the stomach, and other areas of the gut wall, and correlation with function.

Similar to other important contributions to the literature, the observations reported in this paper raise a number of interesting questions to be addressed in future studies.

\section{THE ANIMAL MODEL}

The availability of transgenic mouse models in which specific genes and their products are 'knocked out' has led to numerous important observations. However, mice are well known to exhibit different responses depending on the strain of the mouse and in comparative studies with other species. ${ }^{12,13}$ This places responsibility on the investigator to validate the measurements of the relevant biomarkers in the specific mouse strain being evaluated and correlate these observations with relevant markers of function. It also raises the issue of how generalizable the observations are between species. For example, Choi et al. observed that the volumes of myenteric c-Kit immunoreactive networks of ICC from $\mathrm{nNOS}^{-/}$ mice were significantly decreased compared with control mice. ICC volumes were either decreased or unchanged in the circular muscle layer after normalization for volume of circular smooth muscle, and no significant change in the volume of ICC were observed in the longitudinal layer. In contrast, Iwasaki et al. observed a loss of c-Kit and nNOS expression in the diabetic human antrum that was limited to the circular muscle layer and did not appear to involve the myenteric plexus. ${ }^{14}$ Thus, the general observation that decreased c-Kit immunoreactivity (ICC) is noted in the presence of reduced nNOS expression holds but the specific areas of involvement may be different either because of the disease process or species differences. It is noteworthy that the $\mathrm{nNOS}^{-/-}$mouse model demonstrates an increase in smooth muscle thickness through out the stomach which raises the issue of a 'dilutional' effect with regard to the volumetric measurements of ICC. The authors suggest that this is not the case because changes in c-Kit immunoreactive ICC were greater for ICC-myenteric plexus than ICC-circular muscle, e.g. it would be anticipated that a dilutional effect would be more detectable in the circular muscle layer and this was not the case. The increase in thickness of the muscle layer is thought to be related to the proliferation of smooth muscle cells that is observed in vascular smooth muscle when NO availability is decreased. ${ }^{15}$ It is also relevant that conventional knock out models sometimes produce unexpected results because of inherent lethality that occurs with loss of a particular gene product or a tendency to obscure important modulatory effects of the targeted gene, possibly because of 'adaptive' 
responses that develop in these chronic models. This has led to development of conditional knock out models in which the targeted gene can be inhibited under well-defined conditions established by the investigator. The Cre-LoxP model has emerged as a powerful technology for spatial and temporal control genetic inactivation. ${ }^{16}$

\section{THE MECHANISM(S) OF NO-MEDIATED PROSURVIVAL ON ICC NETWORKS IN THE MOUSE STOMACH}

The authors considered a role for a cytoprotective molecule such as glial derived neurotrophic factor (GDNF) but were dissuaded by observations that GDNF $^{-1-}$ and newborn c-ret ${ }^{-/-}$mice appear to have largely intact ICC. ${ }^{17-19}$ Other mechanisms may explain the apparent cytoprotective effect of NO including increased cellular proliferation, ${ }^{20}$ decreased cell death, ${ }^{21,22}$ and enhanced cell migration. ${ }^{23} \mathrm{NO}$ appears to increase cellular proliferation through several mechanisms including S-nitrosylation or S-nitroglutathione scavenging free radicals and inhibition of caspase-3, up-regulation of antiapoptotic bcl-2 and up-regulation of PI-3 kinase and via Raf/MEK/ERK signaling pathways that are cGMP-independent. ${ }^{24}$ Other studies suggest that $\mathrm{NO}$ is cytoprotective through a cGMP/PKG-dependent pathway and via PI-3 kinase, MEK, PKA and CaMK. ${ }^{25}$ There is an emerging evidence that peroxynitrite, at low concentrations, affords transient cytoprotection via inhibition of PTEN a dual phosphatase that negatively regulates the phosphatidylinositol 3-kinase (PI3K/Akt signaling pathway important for cell survival), thereby activating the antiapoptotic PI3 kinase/Akt pathway in primary neurons. ${ }^{26}$ Activation of heme oxygenase-1 (HO-1) has also been proposed as a mediator of cytoprotection against NO-induced cytotoxicity. ${ }^{27} \mathrm{HO}-1$ is the ratelimiting enzyme that catalyzes the conversion of heme into biliverdin, endogenous carbon monoxide $(\mathrm{CO})$, and free iron. Forms of hemoxygenase identified, to date, include the inducible HO-1 and constitutive HO-2 and H0-3. Some evidence suggests that the overexpression of HO-1 contributes to pathological iron deposition supporting a detrimental role of HO- $1 .{ }^{28}$ However, a growing body of evidence suggests that HO-1 confers cytoprotection against oxidative stress in a number of models. ${ }^{29-31}$ Postmortem studies conducted in humans have revealed increase in HO-1 protein in association with Alzheimer disease, Parkinson disease and Huntington disease. A number of studies indicate that overexpression of HO-1 decreases the neurotoxininduced cell death in transgenic mice and neuronal cultures supporting a cytoprotective role of HO- $1 .{ }^{27}$ $\mathrm{CO}$ can exert potent anti-inflammatory effects in animal and cell culture models of sepsis, liver and lung injury. ${ }^{27-31}$ The protection afforded by $\mathrm{CO}$ against inflammatory lung injury and liver failure was associated with modulation of inducible nitric oxide synthase. ${ }^{30,31}$ and in human pulp cells, HO-1-induced cGMP conferred cytoprotection against NO-induced cyotoxicity. ${ }^{32}$ Elucidating the mechanism(s) underlying the apparent prosurvival effect of NO on ICC in the stomach will be an important area for future research, including the fundamental question of whether $\mathrm{NO}$ is acting directly or indirectly.

\section{REGION-SPECIFIC DIFFERENCES IN THE EXPRESSION OF ICC NETWORKS IN THE MOUSE STOMACH}

This is an important observation that supports the need for mapping the ICC networks throughout the stomach and, indeed, the rest of the GI tract. While laborious to generate, it is evident that the regional morphometric data on ICC networks will be required to provide the foundation for future studies that focus on the physiological and pathophysiological significance of these observations. It is also quite possible that species differences will be observed in the regional distribution of ICC networks, necessitating a need to validate the mapping of ICC networks in each species.

\section{SUMMARY}

Since its emergence as a major novel modulator of cellular function, elucidating the physiologic and pathophysiologic role(s) of nitric oxide has resulted in numerous paradigm shifting observations in the biomedical sciences including the likelihood that other inorganic gaseous molecules, such as $\mathrm{CO}$, have potentially important roles in physiology and pathophysiology. The paper by Choi et al. in this issue of Neurogastroenterology and Motility provides the foundation that nitric oxide has a prosurvival effect on ICC cells in the mouse stomach and serves as a reminder that there will likely be other 'faces' of nitric oxide's biologic actions to be discovered in the future.

\section{REFERENCES}

1 Pacher P, Beckman JS, Liaudet L. Nitric Oxide and peroxynitrite in health and disease. Physiol Rev 2007; 87: 315-424. 
2 Virag L, Szabo E, Gergely P, Szabo C. Peroxynitriteinduced cytotoxicity: mechanism and opportunities for intervention. Toxicol Lett 2003; 140-141: 113-24.

3 Duncan AJ, Heales SJR. Nitric oxide and neurological disorders. Mol Aspects Med 2005; 26: 67-96.

4 Ciani E, Guidi S, Della Valle G, Perini G, Bartesaghi R, Contestabile A. Nitric oxide protects neuroblastoma cells from apoptosis induced by serum depravation through cAMP-response element-binding protein(CERB) activation. I Biol Chem 2002; 277: 49896-902.

5 Ciani E, Guidi S, Bartesaghi R, Contestabile A. Nitric oxide regulates cGMP-dependent cAMP-responsive element binding protein phosphorylation and Bcl-2 expression in cerebellar neurons: implication for a survival role of nitric oxide. J Neurochem 2002; 1282-9.

6 Fiscus RR. Involvement of cyclic GMP and protein kinase $\mathrm{G}$ in the regulation of apoptosis and survival in neuronal cells. Neurosignals 2002; 11: 175-90.

7 Nagai-Kusuhara A, Nakamura M, Mukuno H, Kanamori A, Negi A, Seigel GM. cAMP-responsive element binding protein mediates a cGMP/protein kinase G-dependent anti-apoptotic signal induced by nitric oxide in retinal neuro-glial progenitor cells. Exp Eye Res 2007; 84: 152-62.

8 Choi KM, Gibbons SJ, Roeder JL et al. Regulation of interstitial cells of Cajal in the mouse gastric body by neuronal nitric oxide. Neurogastroenterol Motil 2007; 19: 585-595.

9 Huizinga JD, Thuneberg L, Klüppel M, Malysz J, Mikkelsen $\mathrm{HB}$, Bernstein A. W/kit gene required for interstitial cells of Cajal and for intestinal pacemaker activity. Nature 1995; 373: 347-9.

10 Burns AJ, Lomax AE, Torihashi S, Sanders KM, Ward SM. Interstitial cells of Cajal mediate inhibitory neurotransmission in the stomach. Proc Natl Acad Sci USA 1996; 93: 12008-13.

11 Mashimo H, Kjellin A, Goyal RK. Gastric stasis in neuronal nitric oxide synthase-deficient knockout mice. Gastroenterol 2000; 119: 766-73.

12 Linder CC. Genetic variables that influence phenotype. ILAR J 2006; 47: 132-40.

13 Mckerlie C. Cause and effect considerations in diagnostic pathology and pathology phenotyping of genetically engineered mice. ILAR J 2006; 47: 156-62.

14 Iwasaki H, Kajimura M, Osawa S et al. A deficiency of gastric interstitial cells of Cajal accompanied by decreased expression of neuronal nitric oxide synthase and substance $\mathrm{P}$ in patients with type 2 diabetes mellitus. J Gastroenterol 2006; 41: 1076-87.

15 Cooke JP. The pivotal role of nitric oxide for vascular health. Can J Cardiol 2004; 20(Suppl. B): 7B-15B.

16 Gaveriaux-Ruff C, Kieffer BL. Conditional gene targeting in the mouse nervous system: Insights into brain function and diseases. Pharmacol Ther 2007; 113: 619-34.

17 Ward SM, Ordög T, Bayguinov JR et al. Development of interstitial cells of Cajal and pacemaking in mice lacking enteric nerves. Gastroenterol 1999; 117: 584-94.
$18 \mathrm{Wu}$ JJ, Rothman TP, Gershon MD. Development of the interstitial cell of Cajal. I Neurosci Res 2000; 59: 384-401.

19 Hagl CI, Holland-Cunz S, Schafer KH. What do knockout models teach us about the enteric nervous system? Eur J Pediatr Surg 2003; 13: 170-5.

20 Papapetropoulos A, Gacia-Cardena G, Madri JA, Sessa WC. Nitric oxide production contributes to the angiogenic properties of vascular endothelial growth factor in human endothelial cells. J Clin Invest 1997; 100: 3131-9.

21 Tuteja N, Chandra M, Tuteja R, Misra MK. Nitric Oxide as a unique Bioactive Signaling Messenger in Physiology and Pathophysiology. I Biomed Biotechnol 2004; 4: 227-37.

22 Contestabile A, Ciani E. Role of nitric oxide in the regulation of neuronal proliferation, survival and differentiation. Neurochem Int 2004; 45: 903-14.

23 Boerth NJ, Dey NB, Cornwell TL, Lincoln TM. Cyclic GMP-dependent protein kinase regulates vascular smooth muscle cell phenotype. I Vasc Res 1997; 34: 245-59.

24 Pervin S, Singh R, Hernandez E, Wu G, Chaudhuri G. Nitric oxide in physiologic concentrations targets the translational machinery to increase the proliferation of human breast cancer cells: involvement of mammalian target of rapamycin/eIF4E pathway. Cancer Res 2007; 67: 289-99.

25 Mejia-Garcia TA, Paes-de-Carvalho R. Nitric oxide regulates cell survival in purified cultures of avian retinal neurons: involvement of multiple transduction pathways. I Neurochem 2007; 100: 382-94.

26 Delgado-Esteban $\mathrm{M}$ et al. Inhibition of PTEN by peroxynitrite activates the phosphoinositide-3-kinase/Akt neuroprotective signaling pathway. I Neurochem 2007; 10: 1471-1483.

27 Ryter SW, Choi AM. Cytoprotective and anti-inflammatory actions of carbon monoxide and in organ injury and sepsis models. Novartis Found Symp 2007; 280: 165-75.

28 Tracz MJ, Alam J, Nath KA. Physiology and pathophysiology of heme: implications for kidney disease. I Am Soc Nephrol 2007; 18: 414-20.

29 Orozco-Ibarra M, Chirino YI, Pedraza-Chaverri J. Role of hemeoxygenase-1 in the neurodegenerative disorders. ReV Neurol 2006; 43: 556-62.

30 Fredenburgh LE, Perrella MA, Mitsialis SA. The Role of heme oxygenase-1 in pulmonary disease. Am J Respir Cell Mol Biol 2007; 36: 158-69.

31 Farombi EO, Surh YJ. Heme oxygenase-1 as a potential therapeutic target for hepatoprotection. I Biochem Mol Biol 2006; 39: 479-91.

32 Min KS, Hwang $\mathrm{YH}, \mathrm{Ju} \mathrm{HJ}$ et al. Heme oxgenase -1 mediates cytoprotection against nitric oxide -induced cytotoxicity via cGMP pathway in human pulp cells. Oral Surg Oral Med Oral Pathol Oral Radiol Endod 2006; 102: 803-8. 